\title{
Cetuximab plus chemotherapy as first-line treatment for metastatic colorectal cancer: Effect of KRAS mutation on treatment efficacy in Taiwanese patients
}

\author{
M. C. CHEN, F. F. CHIANG, H. M. WANG* \\ Division of Colorectal Surgery, Taichung Veterans General Hospital, Taichung, Taiwan \\ *Correspondence: whm@vghtc.gov.tw
}

Received November 9, 2012/ Accepted February 11, 2013

\begin{abstract}
Cetuximab, either alone or in combination with chemotherapy, is approved for treatment of patients with metastatic colorectal cancer (mCRC). We reviewed retrospectively records of 50 patients with mCRC from a single center in Taiwan. All patients had ECOG performance status grade 2, histological diagnosis of advanced CRC based on RECIST criteria, and were given at least three cycles of chemotherapy plus cetuximab. We compared the effectiveness of therapy in patients with wild-type and mutant KRAS genes, assessed the overall response (OR) rate of patients with locally advanced or metastatic non-resectable CRC, and assessed the progression-free survival (PFS) time. The ten patients with KRAS mutations had poorer response rates than the 40 patients with the wild-type KRAS gene. Patients with the wild-type and mutant genes had similar progression free survival (PFS) status and median time to PFS. The median overall survival time was significantly greater in patients with the wild-type gene than in those with the mutant gene $(28.77 \pm 6.43$ months $v s .15 .13 \pm 0.50$ months, $p=0.014)$. Taiwanese patients with mCRC respond better to a cetuximab plus chemotherapy regime if their tumors have the wild-type KRAS gene.
\end{abstract}

Key words: cetuximab, colorectal cancer, irinotecan, KRAS, oxaliplatin, 5-fluorouracil

Colorectal cancer (CRC) is the third most common form of cancer world-wide [1]. There are numerous well-known risk factors for CRC, such as germ-line mutations in the APC gene (leading to familial adenomatous polyposis) or one of the seven HNPCC genes (leading to hereditary nonpolyposis colorectal cancer), diet, tobacco smoking, alcohol consumption, and history of adenomatous polyps [2]. The disease originates from colorectal epithelial cells, typically due to somatic mutations of genes in the Wnt-APC- $\beta$-catenin pathway, most commonly in the APC gene [3]. APC is a tumor suppressor gene that normally prevents accumulation of $\beta$-catenin in the nucleus [3]. Somatic mutations in various other genes have also been implicated in the pathogenesis of CRC [3].

Early diagnosis and treatment of CRC is often curative, and patients with early stage disease, in which the cancer has not spread beyond a simple polyp, can be easily treated by resection during colonoscopy [4]. Patients with advanced metastatic disease have significantly lower survival rates and are treated with surgery (if possible) and one of three chemotherapy regimens: FOLFOX-4 (oxaliplatin/leucovorin/5-fluoruracil, given once every two weeks), FOLFIRI (irinotecan/leucovorin/5fluoruracil, given once every two weeks), or Cetuximab (given once weekly) which may be combined with a chemotherapy regimen [5]. Cetuximab is a chimeric monoclonal antibody that binds to epidermal growth factor receptor (EGFR) and the U.S. Food and Drug Association recently approved its use for treatment of head and neck cancer and CRC [6]. For treatment of patients with mCRC (mCRC) in which the tumors are EGFR-positive ( $~ 82 \%$ of all European patients with stage-IV CRC [7]), intravenous cetuximab may be given in combination with chemotherapy or as a single-agent following chemotherapy treatment failure.

Recent studies in Western countries have reported that cetuximab is more effective in treatment of mCRC if the patient's tumor expresses the wild-type form of KRAS, a GTPase in the Ras family of proto-oncogenes. In particular, the multi-center phase III CRYSTAL study (Cetuximab Combined with Irinotecan in First-Line Therapy for Metastatic Colorectal Cancer) and the phase II OPUS study (Oxaliplatin and Cetuximab in FirstLine Treatment of Metastatic Colorectal Cancer) concluded that cetuximab treatment provided benefit only to mCRC patients whose tumors had the wild-type KRAS gene [8-9]. Thus, the FDA recently revised the indications for cetuximab, and currently recommends its use only for the $\sim 60 \%$ of patients whose 
CRC tumors do not have KRAS mutations in codons 12 or 13 of exon 2 [10]. Two recent studies [11, 12] indicated that addition of cetuximab to chemotherapy seems to benefit patients with KRAS G13D-mutant tumors. KRAS mutations have been also reported elsewhere, but these are rarer $[10,11,13]$. A recent multicenter trial of European mCRC patients indicated that $\sim 64 \%$ of patients had the wild-type KRAS gene, $\sim 36 \%$ had the mutated gene [8], and that only those with the wild-type gene benefitted from cetuximab treatment.

The purpose of the present retrospective observational study was to compare the effectiveness of cetuximab plus chemotherapy as treatment for metastatic colorectal cancer in Taiwanese patients with wild-type and mutant forms (codons 12 or 13) of the KRAS gene. In particular, we assessed the overall response (OR) rate of patients with locally advanced or metastatic non-resectable CRC following treatment with chemotherapy plus cetuximab, and assessed the progressionfree survival time of this new cancer treatment.

\section{Patients and methods}

Characteristics of patients. All 50 enrolled CRC patients were treated and followed from December 2005 to October 2010 at the Taichung Veterans General Hospital (Taichung, Taiwan), were at least 18 years-old, had Eastern Cooperative Oncology Group (ECOG) performance status grade 2, and histological diagnosis of advanced (locally advanced or metastatic, nonresectable) measurable colorectal cancer based on Response Evaluation Criteria in Solid Tumours (RECIST) criteria [14]. These criteria were: complete response (CR), disappearance of target lesion; partial response (PR), 30\% or more decrease in diameter of target lesion; progressive disease (PD), $20 \%$ or more increase in diameter of target lesion; stable disease (SD), insufficient change to qualify as PR or PD.

AJCC staging indicated that 1 patient was in stage I, 1 patient was in stage IIA, 3 patients were in stage IIIB, 3 patients were in stage IIIC, and 42 patients were in stage IV.

Treatment regimens. Enrolled patients had undergone at least three cycles of chemotherapy plus cetuximab as first-line treatment for advanced disease. The FOLFOX-4 or FOLFIRI chemotherapy regimen was used, which previous research has indicated are equally effective in the treatment of advanced CRC [15]. For patients who had radiation therapy, the target lesions were those not irradiated, unless there was progression of these lesions into the irradiated field. All patients were EGFR-positive, as determined by testing with the EGFR pharmDx ${ }^{\text {Tm }}$ Stains kit (Dako, Glostrup, Denmark). The exclusion criteria were: age less than 18 years; non-metastatic disease of histologically

Table 1. Primers for KRAS analyses

\begin{tabular}{ll}
\hline Oligo Name & Oligo primer(5'-3') \\
\hline Exon2 Forward & GAATGGTCCTGCACCAGTAA \\
Exon2 Reverse & GTGTGACATGTTCTAATATAGTCA \\
\hline
\end{tabular}

confirmed adenocarcinoma of the colon or rectum; inadequate hematologic function, neutrophil count, renal function, and liver function. This retrospective study was approved by the Institutional Review Board of Taichung Veterans General Hospital, and the requirement for informed consent was waived.

The first-line treatment regimen for all 50 patients was cetuximab plus chemotherapy; the second-line treatments were cetuximab plus other chemotherapy for 28 patients and chemotherapy alone for 4 patients; the third-line treatments were cetuximab only for 1 patient, cetuximab plus other chemotherapy for 4 patients, and chemotherapy alone for 4 patients. In all cases, cetuximab at a dose of $400 \mathrm{mg} / \mathrm{m}^{2}$ was initially administered by a $120 \mathrm{~min}$ intravenous infusion. The subsequent weekly dosage was $250 \mathrm{mg} / \mathrm{m}^{2}$ over $60 \mathrm{~min}$. Cetuximab was given to all patients with mCRC from 2005 to 2008, but was limited to patients with KRAS wild-type tumors since 2009 due to the change in FDA indications [10].

KRAS mutation analysis. Genomic DNA extraction and the KRAS mutation assay were performed using the TheraScreen ${ }^{\oplus}$ : K-RAS Mutation Kit (DxS Diagnostic Innovations, Qiagen Taiwan, Taiwan), according to the manufacturer's instructions. The mutation status of codons 12 and 13 of exon 2 of the KRAS gene were assessed using PCR clamping and melting curve analysis. Table 1 shows the primers used for KRAS analysis. PCR was performed using the HotStarTaq Master Mix Kit (Qiagen, Valencia, California, USA), according to manufacturer's instructions. All sequencing reactions were performed in forward and reverse directions, and all mutations were confirmed by PCR amplification of an independent DNA isolate.

Statistical analysis. Patient characteristics are presented as numbers and percentages, except for age which is presented as mean and range. A frequency table was used to identify the correlation of KRAS gene status with clinical response by use of Fisher's exact test. Progression free survival (PFS) time and overall survival (OS) time are presented as medians \pm standard errors (SEs) for patients with wild-type and mutated $K R A S$ genes. Kaplan-Meier analysis was also used to compare PFS time and OS time for patients with wild-type and mutant KRAS genes, and a log-rank test was performed to compare these results. All statistical analyses were performed using SPSS 15.0 software (SPSS Inc, Chicago, IL, USA). A $p$-value less than 0.05 was considered statistically significant.

\section{Results}

There were 68 colorectal cancer patients who were initially classified as eligible. Eighteen of these patients were excluded (13 pts received second-line cetuximab, 3 pts had fewer than three cycles of cetuximab plus chemotherapy, and 2 pts had no pathology records) and 50 patients were ultimately enrolled. Table 2 shows the demographic and clinical characteristics of the 50 evaluable patients at enrollment. The average age was 56.4 years (range: 30 to 79 years), there were 27 males and 23 females, and the primary tumor site was the colon in 31 patients $(62 \%)$ and the rectum in 19 patients 
(38\%). Metastasis was present in the liver (30 pts, 60\%), lung (10 pts, 20\%), abdomen (10 pts, $20 \%$ ), lymph nodes (8 pts, $16 \%)$, brain ( $2 \mathrm{pts}, 4 \%$ ), omentum ( $1 \mathrm{pt}, 2 \%)$, and rectum (1 pt, $2 \%)$. All 50 patients were given cetuximab in combination with at least three rounds of chemotherapy (irinotecan, 12 pts; oxaliplatin, 39 pts; 5 -fluorouracil, 49 pts; FOLFOX-4 plus irinotecan, $1 \mathrm{pt}$ ) as first-line treatment. The FOLFOX-4 plus irinotecan regimen (FOLFOXIRI) consisted of irinotecan (165 mg/m² in NS $250 \mathrm{~mL} \mathrm{IV} \mathrm{drip} 2 \mathrm{~h}$ QD every 2 weeks/cycle), leucovorin $\left(200 \mathrm{mg} / \mathrm{m}^{2}\right.$ i.v. over $2 \mathrm{~h}$ ), and 5 -fluorouracil (3200 mg/m² i.v. continuous infusion over $48 \mathrm{~h}$ ).

The observed responses were classified as complete response (10 pts), partial response (17 pts), stable response (8 pts), progressive disease ( $8 \mathrm{pts}$ ), and not assessable (7 pts) (Table 2). Analysis of safety data indicated that the drug was generally well-tolerated, but that skin rash (grade-1, 18 pts; grade-2, 19 pts; grade-3, 9 pts) was relatively common. Neutropenia (13 pts) and anemia ( 9 pts) were the most common adverse events (Table 3 ).

Table 2. Demographic and clinical characteristics of enrolled metastatic colorectal cancer patients $(n=50)$.

\begin{tabular}{lc}
\hline Characteristics & Patients, $\mathrm{n}(\%)$ \\
\hline Age, years & \\
Mean (Range) & $56.4(30-79)$ \\
Sex & \\
Male & $27(54.0)$ \\
Female & $23(46.0)$ \\
Primary site & \\
Colon cancer & $31(62)$ \\
Rectal cancer & $19(38)$ \\
Metastasis site & \\
Liver & $30(60)$ \\
Lung & $10(20)$ \\
Bone & $0(0)$ \\
Abdomen & $10(20)$ \\
Brain & $2(4)$ \\
Lymph nodes & $8(16)$ \\
OMENTUM & $1(2)$ \\
Rectal & $1(2)$ \\
Cetuximab use & \\
First line & $50(100)$ \\
Combined chemotherapy & \\
CPT & $12(24)$ \\
OHP & $39(78)$ \\
5FU & $49(98)$ \\
Other & $1(2)$ \\
Response status & \\
Complete response & $10(20)$ \\
Partial response & $17(34)$ \\
Stable disease & $8(16)$ \\
Progressive disease & $8(16)$ \\
Not assessable & $7(14)$ \\
\hline Datawere reprsente \\
\end{tabular}

Data were represented as $\mathrm{n}(\%)$ except age as mean (Range: min. to max.)
Table 4 shows the association of clinical response and KRAS gene mutation status. Ten patients had KRAS mutations and these patients had an overall response rate (complete response + partial response) of $25 \%$ and a complete response rate of

Table 3. Adverse events of patients treated for metastatic colorectal cancer who had the wild-type and mutant forms of KRAS.

\begin{tabular}{|c|c|c|c|c|}
\hline Adverse Event & Grade & Total & $\begin{array}{l}\text { KRAS } \\
\text { Wild Type }\end{array}$ & $\begin{array}{l}\text { KRAS } \\
\text { Mutant }\end{array}$ \\
\hline $\mathrm{N}$ & & 50 & 40 & 10 \\
\hline No of Subject with AE & & $49(98 \%)$ & $40(100 \%)$ & $9(90 \%)$ \\
\hline \multirow[t]{3}{*}{ Anemia } & Grade 1 & $4(8 \%)$ & $3(8 \%)$ & $1(10 \%)$ \\
\hline & Grade 2 & $5(10 \%)$ & $3(8 \%)$ & $2(20 \%)$ \\
\hline & Grade 3 & $0(0 \%)$ & $0(0 \%)$ & $0(0 \%)$ \\
\hline \multirow[t]{3}{*}{ Diarrhea } & Grade 1 & $1(2 \%)$ & $1(3 \%)$ & $0(0 \%)$ \\
\hline & Grade 2 & $0(0 \%)$ & $0(0 \%)$ & $0(0 \%)$ \\
\hline & Grade 3 & $0(0 \%)$ & $0(0 \%)$ & $0(0 \%)$ \\
\hline \multirow[t]{3}{*}{ Fatigue } & Grade 1 & $1(2 \%)$ & $1(3 \%)$ & $0(0 \%)$ \\
\hline & Grade 2 & $5(10 \%)$ & $4(10 \%)$ & $1(10 \%)$ \\
\hline & Grade 3 & $1(2 \%)$ & $1(3 \%)$ & $0(0 \%)$ \\
\hline \multirow[t]{3}{*}{ Leukopenia } & Grade 1 & $0(0 \%)$ & $0(0 \%)$ & $0(0 \%)$ \\
\hline & Grade 2 & $3(6 \%)$ & $1(3 \%)$ & $2(20 \%)$ \\
\hline & Grade 3 & $1(2 \%)$ & $0(0 \%)$ & $1(10 \%)$ \\
\hline \multirow[t]{3}{*}{ Neutropenia } & Grade 1 & $3(6 \%)$ & $2(5 \%)$ & $1(10 \%)$ \\
\hline & Grade 2 & $7(14 \%)$ & $7(18 \%)$ & $0(0 \%)$ \\
\hline & Grade 3 & $3(6 \%)$ & $2(5 \%)$ & $1(10 \%)$ \\
\hline \multirow[t]{3}{*}{ Pain } & Grade 1 & $0(0 \%)$ & $0(0 \%)$ & $0(0 \%)$ \\
\hline & Grade 2 & $2(4 \%)$ & $2(5 \%)$ & $0(0 \%)$ \\
\hline & Grade 3 & $0(0 \%)$ & $0(0 \%)$ & $0(0 \%)$ \\
\hline
\end{tabular}

Table 4. Association of $K R A S$ gene status with clinical response.

\begin{tabular}{lccc}
\hline & $\begin{array}{c}\text { KRAS (wild- } \\
\text { type }) \\
(\mathrm{n}=40)\end{array}$ & $\begin{array}{c}\text { KRAS (mutation) } \\
(\mathrm{n}=10)\end{array}$ & $P$ \\
\hline Overall response, CR+PR & $25(71.4)$ & $2(25)$ & $\begin{array}{c}0.037 \\
+\end{array}$ \\
CR & $9(25.7)$ & $1(12.5)$ & 0.044 \\
PR & $16(45.7)$ & $1(12.5)$ & + \\
SD & $6(17.2)$ & $2(25)$ & \\
PD & $4(11.4)$ & $4(50)$ & \\
Total & $35(100)$ & $8(100)$ & \\
\hline
\end{tabular}

CR: Complete response, PR: Partial response, SD: Stable disease, PD: Progressive disease

* There were seven out of the 50 patients ( 5 in KRAS no mutation, and 2 in KRAS mutation) were not assessable.

Data were represented as $\mathrm{n}(\%)$ and compared between mutation and no mutation using Fisher's exact test.

${ }^{\dagger} P<0.05$, indicated significantly different between KRAS mutation and no mutation. 
(A)

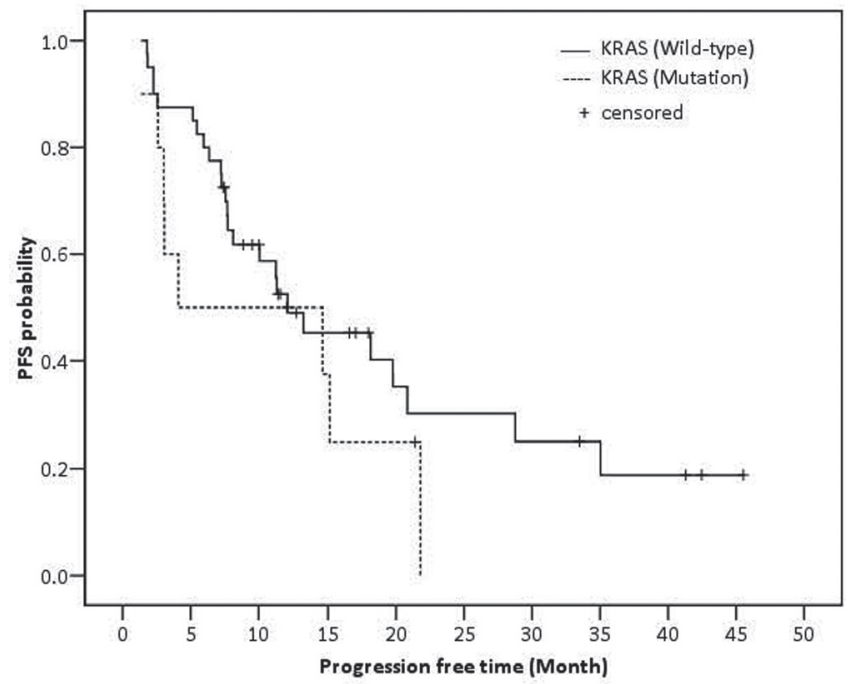

(B)

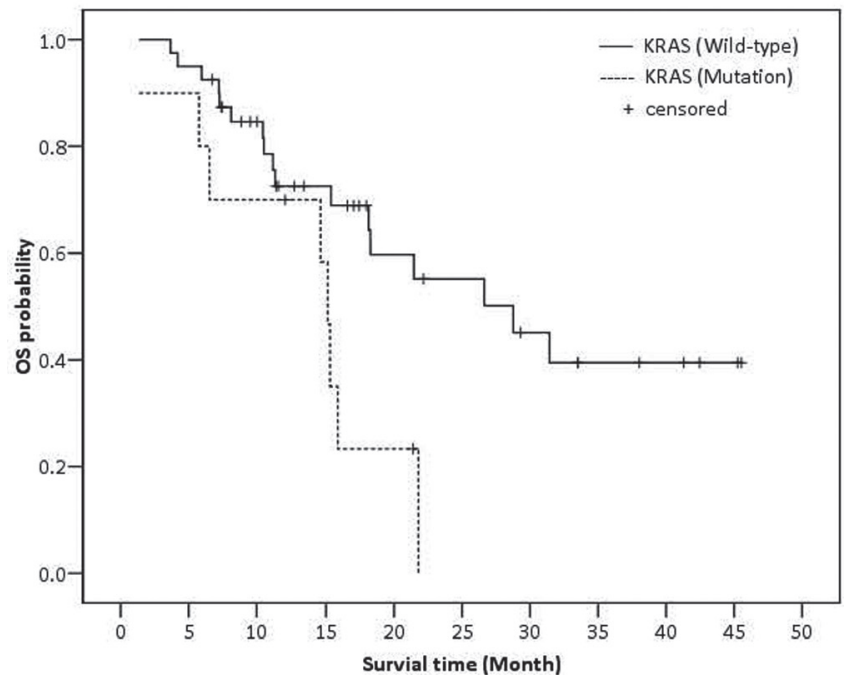

Figure 1. Progression-free survival (PFS) time (A) and overall survival (OS) time (B) of patients with and without the KRAS mutation. Data are presented by the Kaplan-Meier method and compared using a log-rank test. PFS (Fig. A): $p=0.257$, OS (Fig. B): $p=0.014$.

$12.5 \%$. Forty patients had wild-type KRAS genes and these patients had an overall response rate of $71.4 \%$ and a complete response rate of $25.7 \%$. These two differences were statistically significant ( $p=0.037$ and $p=0.044$, respectively), but none of the other comparisons (partial response, stable disease, progressive disease) were significantly different between these two groups.

Figure 1 compares Kaplan-Meier curves for PFS time and OS time for patients with the wild-type and mutant KRAS genes and Table 5 summarizes the resulting statistical com-
Table 5. Summary of the progression-free survival and overall survival of patients with wild-type and mutant forms of the KRAS gene $(\mathbf{n}=\mathbf{5 0})$.

\begin{tabular}{lccc}
\hline & $\begin{array}{c}\text { KRAS (wild-type) } \\
(\mathrm{n}=40)\end{array}$ & $\begin{array}{c}\text { KRAS (mutation) } \\
(\mathrm{n}=10)\end{array}$ & $P$ \\
\hline $\mathrm{N} \mathrm{for} \mathrm{PFS}^{1}$ & $25(62.5 \%)$ & $8(80 \%)$ & 0.461 \\
Median time to PFS, month & $12.13 \pm 3.76$ & $4.10 \pm 8.11$ & 0.257 \\
& & & \\
& & & \\
$\mathrm{~N}$ for OS & & & \\
Median time to OS, month & $28.77 \pm 6.43$ & $15.13 \pm 0.50$ & 0.074 \\
\hline
\end{tabular}

${ }^{1} \mathrm{~N}$ for PFS, number of subjects with progression-free survival status which included subjects with PD or without PD but death.

${ }^{2} \mathrm{~N}$ for OS , number of subjects with overall survival status, that included subjects with death occurred.

Data were represented as $\mathrm{n}(\%)$ in subjects with PFS or OS and compared using Fisher's exact test; whereas time to PFS or time to OS were as shown as estimated median time with standard error (median \pm SE) and compared using Log-rank test.

${ }^{*} P<0.05$, indicated significantly different between KRAS mutation and no mutation.

parisons. Twenty-five patients (62.5\%) with the wild-type gene had PFS status and 8 patients $(80 \%)$ with the mutant gene had PFS status. Fisher's exact test indicated no significant difference between these two groups ( $p=0.461$ ) (Table 5). The median times to PFS were $12.13 \pm 3.76$ weeks for the wild-type group and $4.10 \pm 8.11$ weeks for the mutant group. A log-rank test indicated no significant difference in PFS between these two groups $(p=0.257)$.

Seventeen patients (42.5\%) with the wild-type gene had OS status and 8 patients (80\%) with the mutant gene had OS status. Fisher's exact test indicated no significant difference between these two groups $(p=0.074)$. However, a log-rank test showed that the median time for OS was significantly greater for patients with the wild-type gene than for those with the mutant gene (28.77 \pm 6.43 months $v s .15 .13 \pm 0.50$ months, $p=0.014)($ Table 5, Figure 1).

\section{Discussion}

In the present study, we found that Taiwanese patients with mCRC whose tumors had KRAS mutations in codons 12 or 13 exhibited poorer response to cetuximab plus chemotherapy than patients whose tumors had the wild-type KRAS gene. Although patients whose tumors had the wild-type and mutant KRAS gene had similar PFS and median time to PFS, the median OS time was significantly longer for patients with the wild-type gene. Our results are in agreement with studies of Western patients with mCRC who were treated with cetuximab or panitumumab, another monoclonal antibody that binds to EGFR and is approved for treatment of mCRC [16-19]. Thus, testing the KRAS mutation status of Asian patients with CRC may spare the $\sim 40 \%$ of patients who have mCRC with KRAS mutations from the potential burden of an ineffective treatment. The main novelty of this study is that it is the first study of Asian patients with mCRC to examine the effect of KRAS 
gene status on the effectiveness of cetuximab therapy. Asians and Westerners have different genetic backgrounds, so similar responsiveness would not necessarily be expected.

The KRAS mutation rates in the present study are similar to previously reported rates in Asian and Western populations $[20,21]$. Recent studies reported that patients with G13D mutant tumors benefit more from cetuximab than those with G12V mutant tumors $[11,12]$. In addition, a recent head-tohead comparison of FOLFIRI plus cetuximab versus FOLFIRI plus bevacizumab for mCRC supports the conclusion that KRAS mutations should not be treated as a homogeneous group [22]. Moreover, BRAF, a downstream effector of KRAS, is also mutated some mCRCs and a recent study reported that BRAF tumor mutation $\mathrm{V} 600 \mathrm{E}$ is associated with poor prognosis [21]. Taken together, this suggests that future studies should consider stratification by type of KRAS mutation and $B R A F$ mutation status. Our sample size was too small for meaningful subgroup comparisons.

Identification of biomarkers is becoming increasingly important for the selection of treatments for numerous cancers, including CRC [23]. The initial clinical trials of cetuximab enrolled patients with EGFR-positive tumors, but the results indicated that survival and tumor response were unrelated to EGFR protein expression [24]. This was surprising, given that cetuximab was engineered to bind to EGFR. Intriguingly, an initial study of 110 patients with mCRC indicated that the presence of high levels of epiregulin and amphiregulin (endogenous EGFR ligands) in CRC tumors correlated with response to cetuximab [19]. Thus, determination of KRAS gene status and measurement of epiregulin and amphiregulin allows identification of mCRC patients who best respond to cetuximab therapy. There is a clear need for the identification of additional biomarkers so that patients can be given targeted treatments with known effectiveness rather than non-targeted treatments, which may be futile or even harmful for certain patients [25]. Laurent-Puig et al. [26] reported that lack of PTEN expression was associated with shorter OS in patients with mCRC. However, this study only enrolled patients who received anti-EGFR therapy, with no chemotherapy-only controls. Lack of PTEN expression is probably a poor prognostic factor regardless of anti-EGFR treatment, so the role of PTEN loss in the efficacy of anti-EGFR therapy needs further study.

Many methods can be used to measure KRAS mutations, and each has its strengths and weaknesses [20]. According to the guidelines of the National Comprehensive Cancer Network and the College of American Physicians, real-time PCR and direct sequencing analysis, which we employed here, are two commonly used methods for evaluation of KRAS mutations. Direct sequence analysis has lower analytical sensitivity than some real-time PCR assays [27], but the clinical relevance of a small number cells with mutant KRAS has not been established.

Our results confirm the efficacy of cetuximab plus chemotherapy for treatment of $\mathrm{mCRC}$ in patients whose tumors have the wild-type KRAS gene and also confirm that KRAS gene mutation status of the CRC tumor is an effective biomarker for Asian patients with mCRC. These findings may establish the basis for development of new therapies or biomarkers for metastatic non-resectable colorectal cancer. In particular, given the importance of the status of the KRAS gene for cetuximab therapy, it seems possible that other genes whose proteins are known to interact with the KRAS protein, such as C-raf [28] or RALGDS [29], may also be useful as biomarkers or as therapeutic targets.

The present study has certain limitations. First, this was a retrospective study, so is subject to error due to confounding and selection bias. Although prospective studies are typically required for drug approval, retrospective studies (in the present case, a post-hoc re-evaluation of data), may be acceptable for identification of biomarkers given the fulfillment of certain conditions [10]. Second, our sample size was relatively small, and this may have also led to bias and confounding. Nonetheless, our results are in general agreement with several other retrospective studies that examined the role of KRAS gene status on responsiveness to cetuximab. Thus, we believe that bias and confounding were minimal in the present study. Third, 40 of our patients had KRAS wild-type tumors, but only 10 patients had KRAS mutant tumors, and the unequal sample sizes may have led to bias. However, our use of unequal sample sizes was unavoidable because tumors with wild-type KRAS are significantly more common than those with KRAS mutations, and because cetuximab was initially indicated for all patients with $\mathrm{mCRC}$, but in late 2008 was limited to patients with KRAS wild-type tumors.

In conclusion, our results indicate that Taiwanese patients with metastatic colorectal cancer whose tumors have the wildtype KRAS gene respond better to treatment with cetuximab plus chemotherapy than patients whose tumors have a KRAS gene with a mutation in codons 12 or 13. This information should help Asian clinicians in selecting treatments for patients who have mCRC. Identification of additional CRC biomarkers may allow cetuximab therapy to be targeted for an even more specific group of CRC patients.

\section{References}

[1] GELLAD ZF, PROVENZALE D. Colorectal cancer: national and international perspective on the burden of disease and public health impact. Gastroenterology 2010; 138: 2177-2790. http://dx.doi.org/10.1053/i.gastro.2010.01.056

[2] CHAN AT, GIOVANNUCCI EL. Primary prevention of colorectal cancer. Gastroenterology 2010; 138: 2029-2043. http://dx.doi.org/10.1053/j.gastro.2010.01.057

[3] MARKOWITZ SD, BERTAGNOLLI MM. Molecular basis of colorectal cancer. N Eng J Med 2009; 361: 2449-2460. http://dx.doi.org/10.1056/NEJMra0804588

[4] REX DK, EID E. Considerations regarding the present and future roles of colonoscopy in colorectal cancer prevention. Clin Gastroenterol Hepatol 2008; 6: 506-514. http://dx.doi. org/10.1016/j.cgh.2008.02.025 
[5] GIUliAni F, DE VITA F, COLUCCI G, PISCONTI S. Maintenance therapy in colon cancer. Cancer Treat Rev 2010; 36(Suppl 3): S42-45. http://dx.doi.org/10.1016/S03057372(10)70019-0

[6] BOUCHE O, BERETTA GD, ALFONSO PG, GEISSLER M. The role of anti-epidermal growth factor receptor monoclonal antibody monotherapy in the treatment of metastatic colorectal cancer. Cancer Treat Rev 2010; 36(Suppl 1): S1-10. http://dx.doi.org/10.1016/S0305-7372(10)00036-8

[7] CUNNINGHAM D, HUMBLET Y, SIENA S, KHAYAT D, BLEIBERG $\mathrm{H}$, et al. Cetuximab monotherapy and cetuximab plus irinotecan in irinotecan-refractory metastatic colorectal cancer. N Engl J Med 2004; 351: 337-345. http://dx.doi. org/10.1056/NEJMoa033025

[8] VAN CUTSEM E, KÖHNE CH, HITRE E, ZALUSKI J, CHANG CHIEN CR, et al. Cetuximab and chemotherapy as initial treatment for metastatic colorectal cancer. N Engl J Med 2009; 360: 1408-1417. http://dx.doi.org/10.1056/ NEJMoa0805019

[9] BOKEMEYER C, BONDARENKO I, MAKHSON A, HARTMANN JT, APARICIO J, et al. Fluorouracil, leucovorin, and oxaliplatin with and without cetuximab in the first-line treatment of metastatic colorectal cancer. J Clin Oncol 2009; 27: 663-671. http://dx.doi.org/10.1200/JCO.2008.20.8397

[10] MACK GS. FDA holds court on post hoc data linking KRAS status to drug response. Nat Biotechnol 2009; 27: 110-112. http://dx.doi.org/10.1038/nbt0209-110c

[11] TEJPAR S, CELIK I, SCHLICHTING M, SARTORIUS U, BOKEMEYER C, et al. Association of KRAS G13D tumor mutations with outcome in patients with metastatic colorectal cancer treated with first-line chemotherapy with or without cetuximab. J Clin Oncol 2012; 30: 3570-7. http://dx.doi. org/10.1200/JCO.2012.42.2592

[12] DE ROOCK W, JONKER DJ, DI NICOLANTONIO F, SARTORE-BIANCHI A, TU D, et al. Association of KRAS p. G13D mutation with outcome in patients with chemotherapy-refractory metastatic colorectal cancer treated with cetuximab. JAMA 2010; 304: 1812-20. http://dx.doi. org/10.1001/jama.2010.1535

[13] LOUPAKIS F, RUZZO A, CREMOLINI C, VINCENZI B, SALVATORE L, et al. KRAS codon 61, 146 and BRAF mutations predict resistance to cetuximab plus irinotecan in KRAS codon 12 and 13 wild-type metastatic colorectal cancer. Br J Cancer 2009; 101: 715-21. http://dx.doi.org/10.1038/sj.bjc.6605177

[14] THERASSE P, ARBUCK SG, EISENHAUER EA, WANDERS J, KAPLAN RS, et al. New guidelines to evaluate the response to treatment in solid tumors. European Organization for Research and Treatment of Cancer, National Cancer Institute of the United States, National Cancer Institute of Canada. J Natl Cancer Inst 2000; 92: 205-16. http://dx.doi.org/10.1093/ jnci/92.3.205

[15] COLUCCI G, GEBBiA V, PAOLETTI G, GIULIANI F, CARUSO M, et al. Phase III randomized trial of FOLFIRI versus FOLFOX4 in the treatment of advanced colorectal cancer: a multicenter study of the Gruppo Oncologico Dell'Italia Meridionale. J Clin Oncol 2005; 23: 4866-4875. http://dx.doi. org/10.1200/JCO.2005.07.113
[16] MORONI M, VERONESE S, BENVENUTI S, MARRAPESE G, SARTORE-BIANCHI A, et al. Gene copy number for epidermal growth factor receptor (EGFR) and clinical response to antiEGFR treatment in colorectal cancer: a cohort study. Lancet Oncol 2005; 6: 279-286. http://dx.doi.org/10.1016/ $\underline{\text { S1470-2045(05)70102-9 }}$

[17] BENVENUTI S, SARTORE-BIANCHI A, DI NICOLANTONIO F, ZANON C, MORONI M, et al. Oncogenic activation of the RAS/RAF signaling pathway impairs the response of metastatic colorectal cancers to anti-epidermal growth factor receptor antibody therapies. Cancer Res 2007; 67: 2643-2648. http://dx.doi.org/10.1158/0008-5472.CAN-06-4158

[18] DE ROOCK W, DE SCHUTTER J, DE HERTOGH G, JANSSENS M, BIESMANS B, et al. KRAS mutations preclude tumor shrinkage of colorectal cancers treated with cetuximab. J Clin Oncol 2007; 25(18S): 4132.

[19] KHAMBATA-FORD S, GARRETT CR, MEROPOL NJ, BASIK M, HARBISON CT, et al. Expression of epiregulin and amphiregulin and $\mathrm{K}$-ras mutation status predict disease control in metastatic colorectal cancer patients treated with cetuximab. J Clin Oncol 2007; 25: 3230-3237. http://dx.doi. org/10.1200/JCO.2006.10.5437

[20] WHITEHALL V, TRAN K, UMAPATHY A, GRIEU F, HEWITT C, et al. A multicenter blinded study to evaluate KRAS mutation testing methodologies in the clinical setting. J Mol Diagn 2009; 11: 543-52. http://dx.doi.org/10.2353/ jmoldx.2009.090057

[21] VAN CUTSEM E, KOHNE CH, LANG I, FOLPRECHT G, NOWACKI MP, et al. Cetuximab plus irinotecan, fluorouracil, and leucovorin as first-line treatment for metastatic colorectal cancer: updated analysis of overall survival according to tumor KRAS and BRAF mutation status. J Clin Oncol 2011; 29: 2011-9. http://dx.doi.org/10.1200/JCO.2010.33.5091

[22] STINTZING S, FISCHER VON WEIKERSTHAL L, DECKER T, VEHLING-KAISER U, JAGER E, HEINTGES T, et al. FOLFIRI plus cetuximab versus FOLFIRI plus bevacizumab as first-line treatment for patients with metastatic colorectal cancer-subgroup analysis of patients with KRAS: mutated tumours in the randomised German AIO study KRK-0306. Ann Oncol 2012; 23: 1693-9. http://dx.doi.org/10.1093/annonc/mdr571

[23] ASGHAR U, HAWKES E, CUNNINGHAM D. Predictive and prognostic biomarkers for targeted therapy in metastatic colorectal cancer. Clin Colorectal Cancer 2010; 9: 274-281. http://dx.doi.org/10.3816/CCC.2010.n.040

[24] LENZ, H-J. Cetuximab in the management of colorectal cancer. Biologics 2007; 1: 77-91.

[25] CHEE CE, SINICROPE FA. Targeted therapeutic agents for colorectal cancer. Gastroenterol Clin North Am 2010; 39: 601-613. http://dx.doi.org/10.1016/i.gtc.2010.08.017

[26] LAURENT-PUIG P, CAYRE A, MANCEAU G, BUC E, BACHET JB, et al. Analysis of PTEN, BRAF, and EGFR status in determining benefit from cetuximab therapy in wild-type KRAS metastatic colon cancer. J Clin Oncol 2009; 27: 5924-30. http://dx.doi.org/10.1200/JCO.2008.21.6796

[27] DIFIORE F, BLANCHARD F, CHARBONNIER F, LE PESSOT F, LAMY A, et al. Clinical relevance of KRAS mutation detec- 
tion in metastatic colorectal cancer treated by Cetuximab plus chemotherapy. Br J Cancer 2007; 96: 1166-9. http://dx.doi. org/10.1038/sj.bjc. 6603685

[28] LEFEBURE B, CHARBONNIER F, DI FIORE F, TUECH JJ, LE PESSOT F, et al. Prognostic value of circulating mutant DNA in unresectable metastatic colorectal cancer.
Ann Surg 2010; 251: 275-280. http://dx.doi.org/10.1097/ SLA.0b013e3181c35c87

[29] GYORFFY B, SCHAFER R. Biomarkers downstream of RAS: a search for robust transcriptional targets. Curr Cancer Drug Targets 2010; 10: 858-868. http://dx.doi.org/10.2174/ $\underline{156800910793357916}$ 